\title{
Secretory pattern of characteristic glycoprotein species from segments of the rat submandibular gland following methoxamine stimulation
}

\author{
Yoshiki Iwabuchi, Chihiro Aoki and Taizo Masuhara \\ Department of Dental Pharmacology, Nippon Dental University at Niigata, \\ 1-8 Hamaura-cho, Niigata City, Niigata 951, Japan
}

[Accepted for publication: September 17, 1985$]$

\begin{abstract}
Key words: Rat submandibular segments/submandibular saliva/glycoprotein species/salivary flow rate / methoxamine
\end{abstract}

It is recognized that the rat submandibular gland consists of five distinct parenchymal segments, acini, intercalated ducts, granular ducts, striated ducts and excretory ducts ${ }^{1)}$, and the function of these segments are different from each other. These parenchymal components can be separated from salivary glands of the rat following a microdissection method devised by us $^{2}$.

Using these dissociated preparations and electrophoretic techniques, we found that the functional segments of the submandibular gland of intact rats contain several glycoprotein species; some of these have a characteristic response to stimulation and are secreted into submandibular saliva in a different manner depending on the kind of drug, such as, cholinergic agonists ${ }^{3)}$ and $\alpha$ or $\beta$-adrenoceptor agonists ${ }^{4}$.

The present study was designed to observe the secretory pattern of these characteristic glycoprotein species in acini and convoluted granular tubules following stimulation by methoxamine, because such observations have not yet been reported. For this purpose, changes in the salivary flow rate and the composition of glycoprotein species were followed during salivation induced by an $\alpha_{1}$ adrenergic agonist, methoxamine.

\section{Materials and Methods}

\section{Preparation of functional segments}

Three groups, each consisting of 4 male Sprague-Dawley rats, six weeks of age, were used in these studies. Preparation of paren- chymal components from rat submandibular glands followed the method reported previously by us $^{2}$. Rats which are intact or at 30 and $60 \mathrm{~min}$ after intraperitoneal injection of $6 \mathrm{mg} / \mathrm{kg}$ of methoxamine (Mexan., Nippon Shinyaku) were anesthetized with sodium pentobarbital ( $50 \mathrm{mg} / \mathrm{kg}$, i.p.) and perfused via carotid artery with a modified Hank's solution containing $0.1 \%$ collagenase, $1.0 \mathrm{mM} \mathrm{CaCl}_{2}$ and $0.1 \%$ bovine serum albumin ( $\mathrm{pH}$ 7.4). The unilateral submandibular gland separated from the sublingual glands was immediately removed, sliced, suspended in the same solution, and incubated for $120 \mathrm{~min}$ at $37^{\circ} \mathrm{C}$ under an atmosphere of $95 \% \mathrm{O}_{2}-5 \% \mathrm{CO}_{2}$. The slices were rinsed with ice-cold modified Hank's solution and then microdissected with the help of fine needles under stereomicroscopic observation and each segments of acini and convoluted granular tubules were separatedly collected into siliconized capillary tubes (Drummond Microcaps, $50 \mu \mathrm{l}$ ).

\section{Collection of submandibular saliva}

Seven rats for one group were fasted but given water ad libitum for the 24 hours prior to the collection of submandibular saliva. Each rat was anesthetized with sodium pentobarbital ( $50 \mathrm{mg} / \mathrm{kg}$, i.p.), and excretory ducts of the sublingual gland were ligated after separation from the adherent tissues submandibular gland. The trachea was cannulated with a polyethylene tube (MRC, $1.2 \times 2 \mathrm{~mm})$. Submandibular saliva was then collected from the tip of the ductal cannula 
using a capillary micropipette (Drummond Microcaps, 10 and $20 \mu \mathrm{l}$ ) for periods of 5 min until $60 \mathrm{~min}$ after the onset of salivation stimulated by methoxamine $(6 \mathrm{mg} / \mathrm{kg}$, i.p.) administration. The total volume of saliva per $5 \mathrm{~min}$ was measured and then promptly stored in small ice-cold test tubes at $-20^{\circ} \mathrm{C}$ until use. At the end of collection, the gland were carefully removed and weighed.

\section{SDS-polyacrylamide micro discelectrophore-} sis

Each segment was dissolved in the same volume of $6 \%(\mathrm{w} / \mathrm{v})$ sodium dodecyl sulphate (SDS) solution containing 10\% 2mercaptethanol and heated at $90^{\circ} \mathrm{C}$ for 3 min. The protein content of each sample of saliva and segments was determined by the Lowry method with bovine serum albumin as the standard ${ }^{5}$. One $\mu \mathrm{l}$ of each sample containing $1 \%(\mathrm{w} / \mathrm{v})$ SDS, $5 \%$ 2-mercaptoethanol and $20 \%$ glycerol was applied on a $10 \mu$ l-capillary packed $4-40 \%$ continuous gradient polyacrylamide gel as described by Rüchel et al. ${ }^{6)}$ Electrophoresis was carried out at $60 \mathrm{~V}$ for $60 \mathrm{~min}$ using $50 \mathrm{mM}$ trisglycine buffer $(\mathrm{pH} 8.4)$ in $0.1 \%$ SDS. The apparent molecular weight of glycoproteins detected on the densitogram was estimated using myosin, $\beta$-galactosidase, phosphorylase b, albumin, ovalbumin, carbonic anhydrase, trypsin inhibitor and $\alpha$-lactalbumin as molecular weight markers. Gels were stained either with $0.2 \%$ Coomassie Brilliant Blue R-250 for protein or periodic-schiff-reagent for glycoprotein. The stained gel was scanned with a Joyce-Loebl 3CS microdensitometer using a filter at $595 \mathrm{~nm}$ for Coomassie Blue and $550 \mathrm{~nm}$ for PAS. The ratio of individual peaks to the total stained area were estimated by weight.

\section{Results and Discussion}

The onset time of the salivation was $14.9 \pm 0.4$ min after methoxamine administration. The time course of the secretory rate and cumulative volume of the submandibular saliva secreted in response to methoxamine is shown in Fig. 1. The secretory rate was maximal at 5 to $10 \mathrm{~min}$ after the onset of

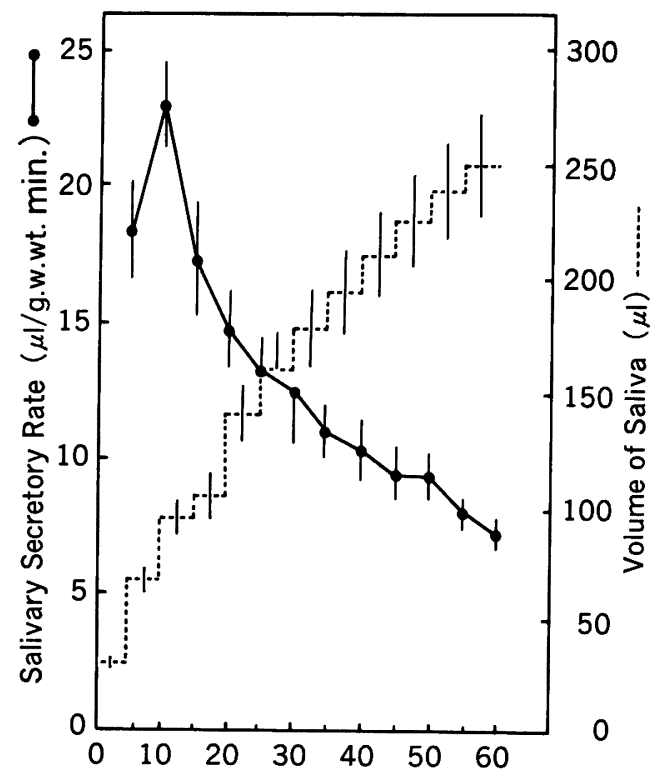

Time After Salivation (min.)

Fig. 1 Time course of secretory rate and accumulative volume of saliva secreted from submandibular glands by methoxamine stimulus. Each point represents the mean \pm S.E. of 7 rats.

salivation and decreased gradually thereafter. The total volume of saliva secreted from the submandibular gland for a 60 min-period was $249.3 \pm 23.0 \mu \mathrm{l}$ per rat.

Although electrophoretic analysis was carried out for all saliva samples collected at 5 min-intervals during salivation after methoxamine stimulus, profile patterns of PASand Coomassie Blue-stained gels applied with submandibular saliva secreted during the 5$10 \mathrm{~min}$ period of salivation, were shown as typical densitograms in Fig. 2. Glycoproteins stained with PAS were migrated on gel as two major bands and three minor bands. One of the two major bands corresponded to band I, which is characteristically found in acini, and the other corresponded to band III characteristically found in convoluted granular tubules, and one of the minor bands was band IV also characteristically found in acini, as previously reported ${ }^{2}$. In the protein profiles stained with Coomassie Blue of the representive sample of Fig. 2(A), 

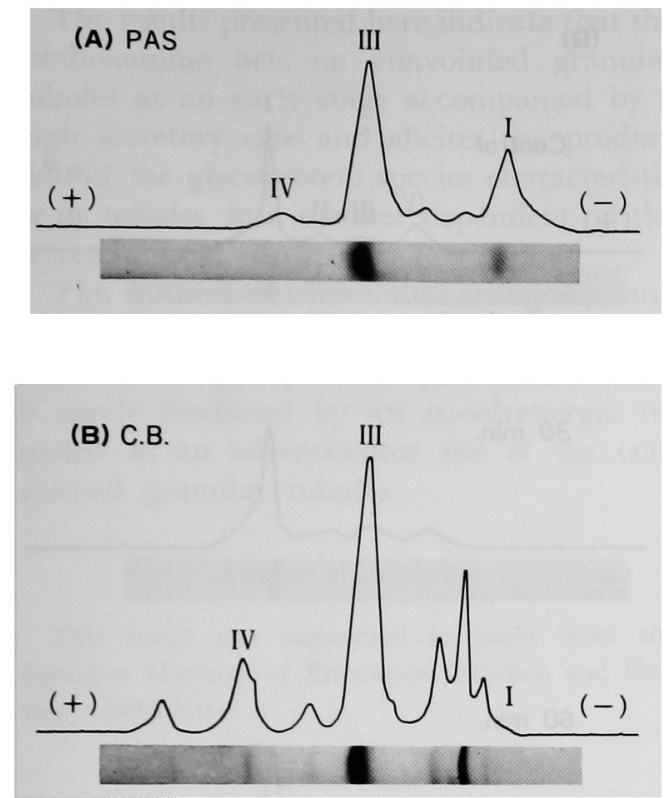

Fig. 2 Typical densitograms of glycoprotein bands (A) and protein bands (B) of submandibular saliva secreted during the 5-10 min period after stimulus of salivation by methoxamine. Samples containing $0.25 \mu \mathrm{g}$ and 0.5 $\mu \mathrm{g}$ as protein were applied on gels for staining protein and glycoprotein, respectively.

protein bands were 7 to 8 in number as shown in Fig. 2(B). The peak of the protein band corresponding to the glycoprotein band III was significantly higher than that of the protein band corresponding to glycoprotein band I as shown in Fig. 2(B). These results, therefore, suggest that the carbohydrate moiety is higher in band I than in band III. Similarly, the carbohydrate moiety was higher in band I than in band IV. Molecular weights of the protein bands corresponding to the glycoprotein band I, III and IV were approximately $130 \mathrm{~K}, 31 \mathrm{~K}$ and $21.5 \mathrm{~K}$ dalton, respectively, the same as previously reported $^{2)}$.

The time course changes of relative amounts of each main band which was collected at periodic intervals of $5 \mathrm{~min}$ was shown in Fig. 3. Relative amounts of glycoprotein band III increased at the early stage and reached the maximum during the 10-

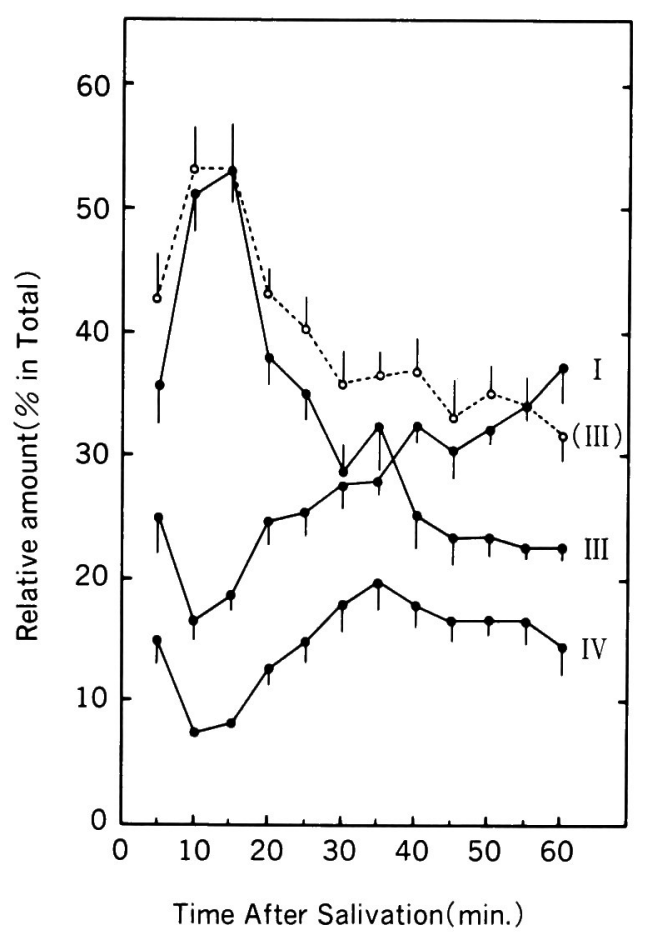

Fig. 3 Time course of relative amounts of glycoprotein and protein bands in saliva secreted from submandibular glands by methoxamine stimulus. - glycoprotein band, O---O protein band. Each point represents the mean \pm S.E. of 7 rats.

$15 \mathrm{~min}$ period after the onset of salivation and decreased rapidly thereafter. This curve of band III was almost parallel with the change in the flow rate of secretion. On the other hand, the relative amount of band I decreased to the lowest level during the 5-10 min period and increased gradually thereafter. The initial values of the relative amounts of band I and III plotted on the figure may partially reflect relative amounts of band I and III from the resting saliva filling ducts of the gland.

Based on the proof ${ }^{4}$ that the secretion into the saliva of glycoprotein species of band III is derived mainly from convoluted granular tubules when elicited by methoxamine stimulus, an electrophoretic investigation was tried on two parenchymal segments of acini and convoluted granular tubules 
(A)

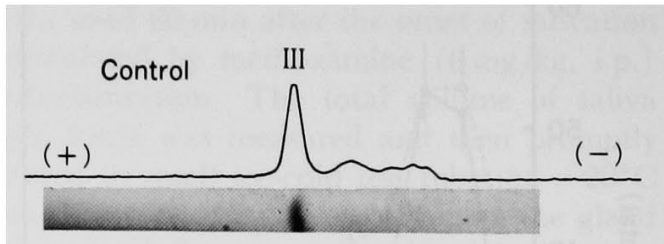

$30 \mathrm{~min}$.

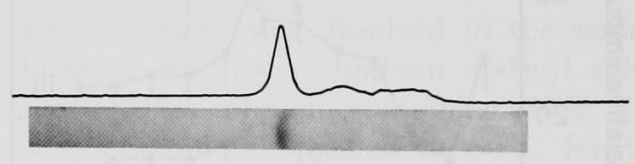

$60 \mathrm{~min}$.

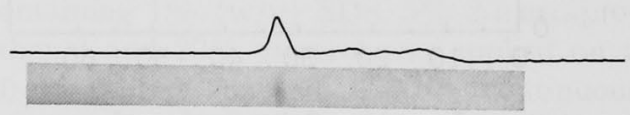

Fig. 4(A) Densitograms of glycoprotein bands of convoluted granular tubules at 0 , 30 and $60 \mathrm{~min}$ after methoxamine administration. Samples containing $1 \mu \mathrm{g}$ as protein were applied on gels.

which were isolated at 30 and $60 \mathrm{~min}$ after methoxamine administration. The results obtained are shown in Fig. 4. In the convolute granular tubules, as shown in Fig. 4(A), the glycoprotein species of band III was reduced markedly $60 \mathrm{~min}$ after methoxamine administration. Whereas, in the acini, glycoprotein species of band I did not show any appreciable change at $30 \mathrm{~min}$, but it showed a little reduction at $60 \mathrm{~min}$ as shown in Fig. 4(B).

As a result of electrophoretic analyses of stimulated submandibular saliva in rats ${ }^{2}$, it has been postulated that the characteristic proteins ( $\alpha$-type) secreted in response to high doses of $\alpha$-adrenergic agonist could be derived mainly from the convoluted granular tubules, whereas those ( $\beta$-type) elicited by $\beta$-adrenergic and cholinergic agonists and low doses of $\alpha$-adrenergic agonist were primarily from the acinar cells. Tsukutani ${ }^{8)}$
(B)
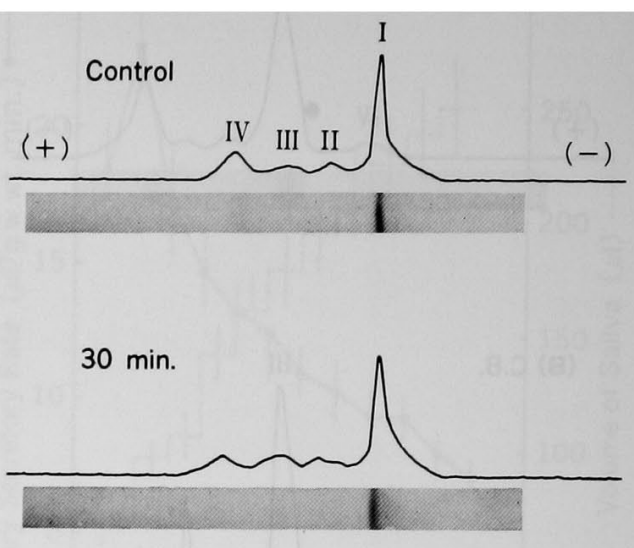

$60 \mathrm{~min}$.

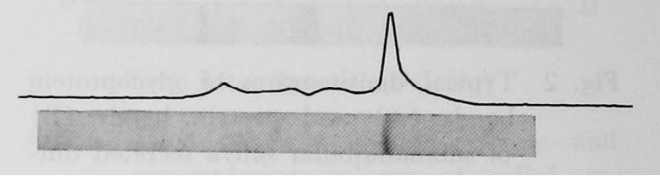

Fig. 4(B) Densitograms of glycoprotein bands of acinus obtained at 0,30 and 60 min after methoxamine administration. Samples containing $1 \mu \mathrm{g}$ as protein were applied on gels. recently described that $15 \mathrm{mg} / \mathrm{kg}$ of methoxamine stimulated the convoluted granular tubules of the submandibular glands in mice and initiated both morphological changes of the cells and degranulation of secretory granules contained in the cells about 20-30 min after the drug administration, and the extent of the degranulation increased with time. The onset time of degranulation, judged by the increase of glycoprotein band III in secreted saliva in this study, was about $20 \mathrm{~min}$ after methoxamine administration and agreed with the above histochemical findings in mice. Although our methods and experimental animals differ from Tsukutani et al. ${ }^{8}$, concurrence of the onset time would indicate that secretory response of convoluted granular cells to methoxamine took place at the early stage of stimulation, and may produce concomitant degradation of secretory granules, 
The results presented here indicate that the methoxamine acts on convoluted granular tubules at an early stage accompanied by a high secretory rate and elicites the production of the glycoprotein species characteristic in its tubules, in a manner dependent on the secretory rate.

The authors consider that using $\alpha_{1^{-}}$and $\alpha_{2}$-blocking agents, may confirm whether the secretion of glycoprotein species, band III, is surely mediated by an $\alpha_{1}$-adrenergic response at an adrenoceptor site of the convoluted granular tubules.

\section{Acknowledgements}

This study was supported in parts from the Japanese Ministry of Education, Science and Culture $(60480404)$.

\section{References}

1) Tamarin, A. and Sreebny, L. M.: The rat submandibular salivary gland. A correlative study by light and electron microscopy. J. Morphol. 117: 295-352, 1965.

2) Masuhara, T. and Iwabuchi, Y.: Microdiscelectrophoretic study on glycoproteins of parenchymal components dissociated from rat submandibular gland. Jpn. J. Oral Biol. 25:
1177-1180, 1983.

3) Masuhara, T. and Iwabuchi, Y.: Characterization of glycoproteins in submandibular acinus and saliva of rat elicited by pilocarpine. Jpn. J. Oral Biol. 26: 280-283, 1984.

4) Iwabuchi, Y., Aoki, C. and Masuhara, T.: Effects of adrenergic agents on glycoprotein secretion from acini and granular convoluted tubules of the rat submandibular gland. Jpn. J. Oral Biol. 27: 741-745, 1985.

5) Lowry, O. H., Rosebrough, N. J., Farr, A. L. and Randall, R. J.: Protein measurement with the Folin phenol reagent. J. Biol. Chem. 193 : 265-275, 1951.

6) Rüchel, R., Mesecke, S., Wolfrum, D. I. and Neuhoff, V.: Mikroelektrophorese an kontinuierlichen polyacrylamide Gladiented Gelen. I. Herstellung und Eigenschaften von Gelgradienten in Kapillaren; ihre Anwendung zur proteinfraktionierung und Molgewichtsbestimmung. Hoppe-Seyler's Z. Physiol. Chem. 354: 1351-1368, 1973.

7) Abe, K. and Dawes, C.: The effects of electrical and pharmacological stimulation on the types of proteins secreted by rat parotid and submandibular glands. Arch. Oral Biol. 23: 367-372, 1978.

8) Tsukutani, K.: Immunohistochemical studies in granular convoluted tubules of mice submandibular glands. Jpn. J. Oral Biol. 27 : 569-594, 1985 (in Japanese). 